\title{
Antitumoral and antioxidant activities of lipophilic and phenolic extracts from Cynara cardunculus L. var. altilis (DC)
}

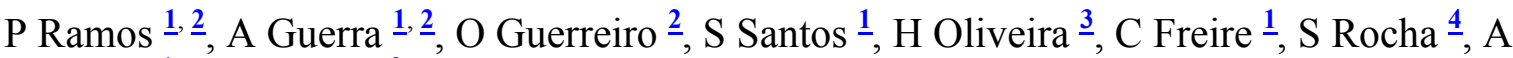 \\ Silvestre $\stackrel{1}{ }$, MF Duarte $\stackrel{2}{-}$
}

- $\quad{ }^{1}$ CICECO and Department of Chemistry, Campus Universitário de Santiago, University of Aveiro, 3810 - 193 Aveiro, Portugal

- ${ }^{2}$ Centro de Biotecnologia Agrícola e Agro-Alimentar do Alentejo (CEBAL)/Instituto Politécnico de Beja (IPBeja), Rua Pedro Soares s.n. - Campus IPBeja/ESAB, Apartado 6158, 7801 - 908 Beja, Portugal

- ${ }^{3}$ CESAM \& Department of Biology, Laboratory of Biotechnology and Cytometry, Campus Universitário de Santiago, University of Aveiro, 3810 - 193 Aveiro, Portugal

- ${ }^{4}$ QOPNA and Department of Chemistry, Campus Universitário de Santiago, University of Aveiro, 3810 - 193 Aveiro, Portugal

\section{- $\quad \underline{\text { Congress Abstract }}$}

Cynara cardunculus L. var. altilis (DC) (Cca) grows under the semi-arid conditions as those of south of Portugal. Given to high cellulose and hemicelluloses contents, paper pulp production has been proposed [1]. Moreover, the extraction of bioactive compounds could also be considered for an integrated valorization of Cca. Sesquiterpene lactones (SL) and pentacyclic triterpenes, the major constituents of Cca leaves and capitula lipophilic fractions [2] showed lower tryglyceride levels in rat serum [3] and anti-inflammatory [4] actions, respectively. Besides, cardoon extracts displayed antioxidant [5] and antitumor properties [6, 7], mainly due to hydroxycinnamic acid derivatives. The present work aims to determine the chemical composition of Cca lipophilic and phenolic fractions, by analyzing respectively their dichloromethane (DCM) and methanol/water/acetic acid (MWAA) (49.5:49.5:1) extracts, by gas chromatography and high temperature-ultra-high pressure liquid chromatography coupled with mass spectrometry. Furthermore, the antitumoral activity of Cca DCM extracts was evaluated on human breast cancer cells (MDA-MB-231), by assessing cell proliferation $(2-500 \mu \mathrm{g} / \mathrm{mL})$, cell cycle and Akt molecular signaling $(10.4 \mu \mathrm{g} / \mathrm{mL})$. Additionally, the antioxidant activity of MWAA extracts $(7.5-400 \mu \mathrm{g} / \mathrm{mL})$ was determined by DPPH scavenging assay. Cca leaves DCM extract, containing $48.5 \pm 3.1 \%$ SLs (Table 1 ), and the major compound, cynaropicrin (2), strongly inhibited MDA-MB-231 cellular viability, inducing cell cycle arrest at G2 phase, with inhibition of Akt phosphorylation at serine 473. In addition, MWAA extracts from Cca stalks outer part, containing $5.9 \pm 0.5 \%$ hydroxycinnamic acids (HA), were the most effective to scavenge DPPH free radicals. The antioxidant activity was correlated to phenolic $(\mathrm{r}=-0.897)$ and HAs $(\mathrm{r}=-0.990)$ contents. In conclusion, Cca leaves and stalks outer part are valuable sources of respectively SLs and HAs, with a great antitumoral and antioxidant potential. 


\begin{tabular}{|l|l|}
\multicolumn{2}{|c|}{$\begin{array}{c}\text { Tab. 1: Chemical composition of Cynara cardunculus L. var. atilis (DC) leaves } \\
\text { dichloromethane extract by gas chromatography mass spectrometry analysis. }\end{array}$} \\
\hline Compound & Extract content (\% w/w) \\
\hline Sesquiterpene lactones & $48.5 \pm 3.1$ \\
\hline Cynaropicrin & $45.5 \pm 2.9$ \\
\hline Grosheimin & $2.1 \pm 0.1$ \\
\hline Deacylcynaropicrin & $0.9 \pm 0.1$ \\
\hline Pentacyclic triterpenes & $3.7 \pm 0.2$ \\
\hline Taraxasterol & $1.0 \pm 0.3$ \\
\hline$\Psi$-Taraxasterol & $0.5 \pm 0.2$ \\
\hline Taraxasterol acetate & $0.5 \pm 0.1$ \\
\hline Lupeol & $0.4 \pm 0.2$ \\
\hline$\Psi$-Taraxasterol acetate & $0.4 \pm 0.5$ \\
\hline$\beta$-Amyrin & $0.3 \pm 0.1$ \\
\hline$\beta$-amyrin acetate & $0.3 \pm 0.2$ \\
\hline$\alpha$-Amyrin & $0.1 \pm 0.1$ \\
\hline$\alpha$-amyrin acetate & $0.1 \pm 0.1$ \\
\hline Lupeoyl acetate & $0.1 \pm 0.1$ \\
\hline Sterols & $0.4 \pm 0.2$ \\
\hline Fatty acids & $0.3 \pm 0.0$ \\
\hline Long chain aliphatic alcohols & $0.2 \pm 0.3$ \\
\hline Aromatic compounds & $<0.1$ \\
\hline Others & $0.3 \pm 0.1$ \\
\hline Total & $53.4 \pm 3.3$ \\
\hline
\end{tabular}

\section{References:}

[1] Fernández, J., et al. (2006) Ind. Crops Prod. 24: 222 - 229.

[2] Ramos, P. A. B., et al. (2013)J. Agric. Food Chem. 61: 8420 - 8429.

[3] Shimoda, H., et al. (2003) Bioorg. Med. Chem. Lett. 13: 223 - 228.

[4] Yasukawa, K., et al (2010)J. Nat. Med. 64: 388 - 391.

[5] Valentão, P., et al. (2002)J. Agric. Food Chem. 50: 4989 - 4993. 
[6] Mileo, A. M., et al. (2012)J. Cell. Physiol. 227: 3301 - 3309.

[7] Velez, Z., et al. (2012) Agriculture 2: 472 - 492. 